\title{
Cold water on icosahedral symmetry
}

\section{Linus Pauling has produced an alternative explanation of the observation that solid manganese- aluminium alloy may have 5-fold symmetry on the atomic scale. How can the two views be reconciled?}

$D_{R}$ Linus Pauling, the long-standing subversive, has once more thrown a cat among some pigeons. His article on page 512 of this issue is several things, but not least a reminder that his career began in the $1920 \mathrm{~s}$ as that of a crystallographer. The trouble that Pauling's article will now stir up is simply that it denies the need for speculation about "a new kind of matter" to explain observations of 5-fold rotational symmetry in the structure of solid specimens of a manganese-aluminium alloy.

The first observations were reported at the beginning of last year by Schechtman et al. (for this and other references, see Pauling's article). The alloy, which in composition approximates $\mathrm{MnAl}_{6}$, is made in small quantities by fastquenching molten metal. Most simply, $\mathrm{X}$ ray diffraction in the appropriate directions yields patterns of spots with clear 5 -fold symmetry. The observations have been repeatedly confirmed and ingeniously explained by the notion of quasicrystalline matter. For all practical purposes, Pauling now says that these schemes are unnecessary.

The elementary textbooks carefully explain why the only kinds of rotational symmetry consistent with the translational symmetry of a crystal lattice are those with 2-, 3-, 4- and 6-fold axes. Five-fold symmetry axes are ruled out because if rotation by a fifth of a full circle will leave the crystal lattice unchanged, rotation by twofifths of a full circle will not. But Levine and others have sought to construct threedimensional atomic frameworks for solid materials by a technique recognized, after the event, as a generalization of the Penrose covering of the two-dimensional plane with tiles shaped like one or other of two distinct rhombi (see Nature 313, 263). The Penrose tiling has some strict 5-fold axes of symmetry (of rotation perpendicular to the plane). Moreover, all edges marking the boundaries between tiles have orientations belonging to a finite set of directions in the plane. The price paid for pentagonal symmetry is that strict translational order is lost.

These are hallmarks of the "quasicrystal", as elaborated during the past year. A three-dimensional framework of atoms can be built so as to have true 5 -fold symmetry, because in Penrose tiling neighbour-neighbour bonds have fixed orientation but translational periodicity is replaced by quasi-periodicity. All lattice points are specified as integral combinations of vectors with lengths incommensu- rate with each other. It is generally agreed that the theories are quite fun.

Pauling's approach is classical and sceptical. Why not, instead, see what traditional model building might accomplish? Quite apart from the significance of the result, the argument is an illustration of how intuition, experience and apparently universal knowledge can be used to fashion a complicated and unknown crystal structure out of thin air.

The interdiction against 5-fold symmetry in crystal lattices does not of course imply that atomic arrangements, molecules perhaps, with 5-fold symmetry, cannot form solids but merely that the symmetry of the groupings cannot be imparted to that of the structure as a whole. So Pauling starts with simple structures which do have 5-fold symmetry, the known icosahedral intermetallic complexes such as that of tungsten and aluminium. The 5-fold symmetry of a regular icosahedron is self-evident in that the twelve faces of this regular polyhedron are regular pentagons, but apart from the 5fold axes through each face, there are also 3 -fold axes through each vertex.

The conceptual model-building from this point on depends to some extent on the happy chance that the tetrahedral angle $\left(109.47^{\circ}\right)$ is not very different from the included angle of a regular pentagon. To give the material a chemical composition corresponding to the Schechtman alloy, Pauling assumes each elementary icosahedron shares four faces with others. By bending the angles a little, he shows that five icosahedra can make a ring, or that twenty can yield a structure with the symmetry of a dodecahedron.

For the Schechtmann alloy, what matters most, in Pauling's argument, is that the spots on the diffraction diagrams correspond to those the model predicts. $\mathrm{Re}$ markably, the predicted positions are those determined by the simplest guess about the radii of the cores of manganese and aluminium. That the intensities of the spots should also vary systematically as predicted by the model is a clinching piece of circumstantial evidence.

So is the Schechtman alloy a regular crystal in the Pauling mould. or a quasicrystal as described by Levine and others? There is no doubt that the observations are unshakable evidence of 5-fold symmetry and that the symmetry extends over regions large enough for the diffraction spots to be sharp, which leads to the estimate that the microcrystals responsible have dimensions measured in micrometres. Pauling, on the other hand, has shown how it would be natural for a manganese-aluminium alloy with the Schechtmann composition to form into arrangements which, on the scale of some tens of angstroms, resemble that of a dodecahedron. On Pauling's model, this structure would form a regular crystal with a cubic unit cell of dimensions $26 \AA$. One way of visualizing the result is as a series of concentric shells built up of icosahedra sharing common faces which, because of their inherant trigonal symmetry, are arranged in space relative to each other much as are the carbon atoms in diamond. But then, the argument goes, because of the strain entailed in bending the tetrahedra into pentagonal angles and, more important, the twisting involved in forming chains of icosahedra into close rings, the time comes when it is energetically advantageous to start building a new crystal on each of the twenty faces of the underlying dodecahedron. The result, Pauling says, should be a speck of solid material built from a seed with dimensions of the order of $100 \AA$ supporting up to 20 crystal twins, each with a different orientation. Given the size of the underlying structure, it may not be unreasonable to suppose these structures growing to micrometre dimensions.

So how are the two structures to be reconciled? Pauling has one practical suggestion: more accurate $\mathrm{X}$-ray diffraction measurements with monochromatic $\mathrm{X}$ rays so as to be able to index a much larger number of diffraction spots and then more accurately to compare prediction and observation. If such experiments confirm his ingenious model, it will seem a triumph for the intuitive crystal model-building of the traditional kind.

If the Schechtman alloy is not, after all, an example of a quasi-crystal structure in the sense introduced by Levine. where should people look for realizations of structures of that novel kind, which are not inherently implausible? And what relationship is there between the atomic framework that Pauling has built on paper and that suggested by the advocates of quasi-crystals? It would be a gigantic task to assemble all the atomic coordinates of a crystal in the Pauling mould; might it even be that. if there were done. it would turn out that the two descriptions are but different ways of describing the same kind of arrangement? That would save a lot of trouble. 